- Case Report

\title{
Afatinib-Induced Acute Fatal Pneumonitis in Metastatic Lung Adenocarcinoma
}

\author{
Sang Hoon $\mathrm{Yoo}^{+}$, Jin Ah Ryu${ }^{+}$, Seo Ree Kim, Su Yun Oh, Gu Sung Jung, Dong Jae Lee, Bong Gyu Kwak, \\ Yu Hyun Nam, Kyung Hyun Kim, Young Jun Yang* \\ Department of Internal Medicine, Daejeon St. Mary's Hospital, The Catholic University of Korea College of Medicine, Daejeon, Korea
}

\begin{abstract}
Afatinib is an oral tyrosine kinase inhibitor (TKI) that inhibit Endothelial Growth Factor Receptor (EGFR), Human Epidermal Growth Factor Receptor 2 (HER2), and HER4. The common side effects of EGFR TKI are rash, acne, diarrhea, stomatitis, pruritus, nausea, and loss of appetite. Drug induced pneumonitis is the less common adverse effects of EGFR TKI. Afatinib, 2nd generation EGFR TKI is anticipated to overcome drug resistance from 1st generation EGFR TKI according to preclinical study, and several studies are being conducted to compare clinical efficacy between 1st and 2nd EGFR TKI. Several cases of rug induced acute fatal pneumonitis were reported after use of erlotinib or gefitinib. However, a case of acute fatal pneumonitis associated with afatinib was note reported except drug induced pneumonitis in other clinical study. Here, we present a cases of acute severe pneumonitis related with afatinib in metastatic lung adenocarcinoma with literature review.
\end{abstract}

Keywords: Afatinib; Acute Fatal Pneumonitis; Metastatic Lung Adenocarcinoma 


\section{INTRODUCTION}

Lung cancer is the leading cause of death due to malignancy. It has a poor prognosis and the 5 -year survival rate is only $15 \%$. Most lung cancers are asymptomatic; therefore, the majority of patients are diagnosed with late-stage disease, which restricts treatment options, with the exception of chemotherapy.

However, the identification of EGFR mutations and the introduction of epithelial growth factor receptor-tyrosine kinase inhibitors (EGFRTKIs) have expanded treatment options and improved results. On average, acquired drug resistance to erlotinib and gefitinib, 1st-generation EGFR-TKIs, has been observed between 8 and 16 months of use. ${ }^{1)}$ Afatinib, a 2nd-generation EGFR-TKI, is expected to overcome the acquired resistance that develops with 1st-generation EGFR-TKIs by irreversibly blocking not only EGFR but also human epidermal growth factor receptor 2 (HER2) dimer formation. Currently, there is an ongoing comparative study investigating the efficacy of 1st- and 2nd-generation EGFR-TKIs in lung cancer. In addition to erlotinib and gefitinib, afatinib has been reported to be responsible for drug-induced pneumonitis as a common adverse effect in clinical trials. ${ }^{2,3}$ Several preclinical studies have reported acute drug-induced pneumonitis after erlotinib and gefitinib use, whereas there have been no reports of the same fatal adverse effects with afatinib. In the present case, we emphasize the need for caution with afatinib use as it may result in fatal pulmonary complications.

\section{CASE REPORT}

A 78-year-old woman was admitted for dry cough and weight loss via our outpatient clinic on 8 August 2015. She had been undergoing medical treatment for diabetes, dyslipidemia, and osteoporosis since
20 years. The patient was a social drinker and a nonsmoker. She was diagnosed with metastatic lung adenocarcinoma (T1b N0 Mla, stage IV) with EGFR mutations on the basis of the findings obtained with chest computed tomography (CT), positive emission tomography-CT, percutaneous transthoracic needle aspiration (PCNA) of the left superior lobe, and wedge resection of the right superior lobe (Figures 1, 2). The biopsy results from the PCNA results were obtained on August 22nd and the wedge resection results were obtained on August 28th. Afatinib treatment ( $40 \mathrm{mg} / \mathrm{d}$ ) was initiated on 2 September 2015; no specific complications were observed during afatinib use and the patient was discharged. She was re-admitted for acute dyspnea on 7 September 2015 via the outpatient clinic; she had been consuming afatinib daily for 6 days.

The patient's initial vital signs were as follows: blood pressure, 110/70 mm Hg; heart rate, 100 beats/min; respiratory rate, 20 breaths/ min; body temperature, $37.7^{\circ} \mathrm{C}$; and oxygen saturation, $63 \%$ as room air. On auscultation, coarse breathing sounds and crackles were heard over both the lungs fields. Initial laboratory test results were as follows: total leukocyte count, 10,300/ $\mathrm{mm}^{3}$; hemoglobin, $9.6 \mathrm{~g} / \mathrm{dL}$; platelet count, 217,000/ $\mathrm{mm}^{3}$; C-reactive protein, $18.66 \mathrm{mg} / \mathrm{dL}$; and D-dimer, $1.78 \mathrm{mg}$ fibrinogen equivalent units $/ \mathrm{mL}$. All other parameters were within their respective normal limits.

A chest CT was conducted on the suspicion of pulmonary thromboembolism and drug-induced pneumonitis. It showed that there were new consolidations and ground glass opacity shadows, whereas the size of lung cancer itself had decreased. A pulmonary thromboembolism was not observed (Figure 3 ). The patient was diagnosed with acute severe drug-induced pneumonitis after afatinib use. This diagnosis was based on the following: the onset of symptoms after drug application, the absence of another explanatory cause for the hypoxia, the results of the chest CT, and the negative results of the nasal swab
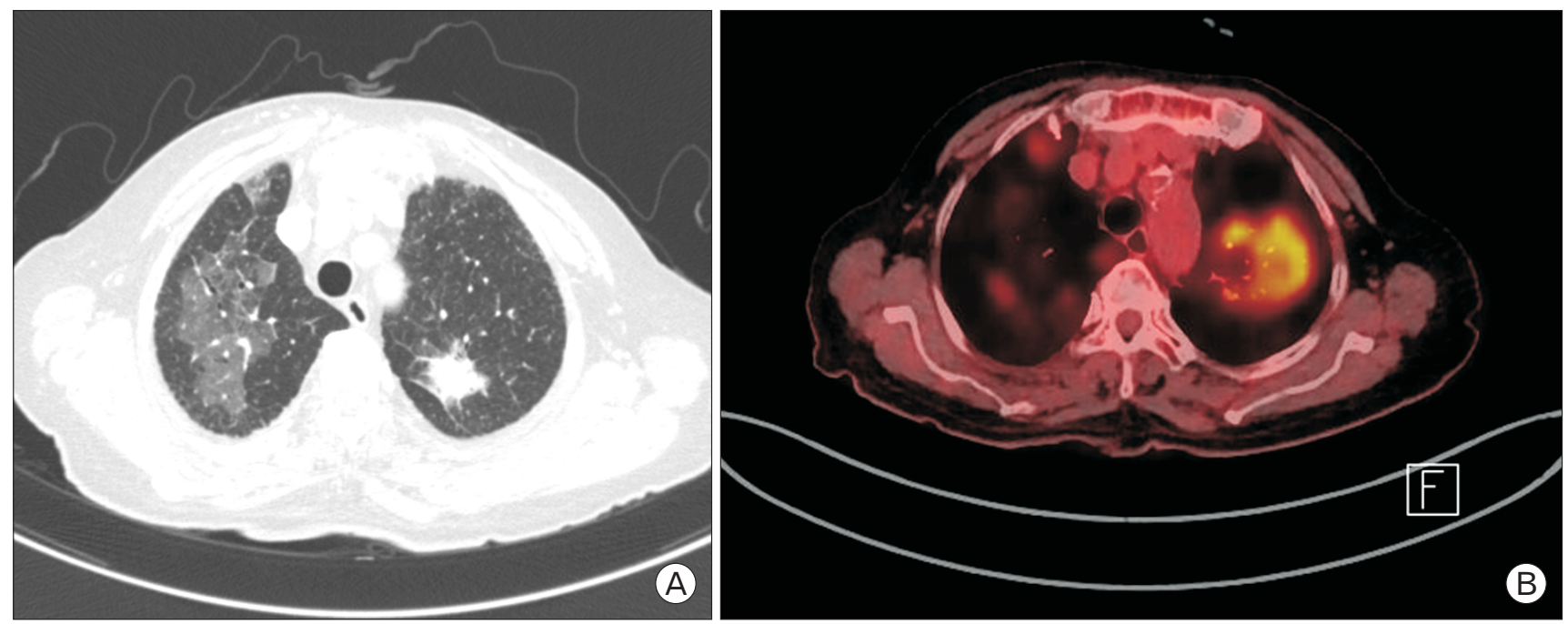

Figure 1. (A) A chest CT scan showing a solid nodule, approximately $2.7 \mathrm{~cm}$ in size, with peripheral GGO, spiculated margins in the left upper lobe, and lobular GGO with some consolidation and mild interlobular septal thickening in the right upper lobe. (B) A positron emission tomography-CT scan showing irregular consolidation with increased uptake in the left upper lobe and active inflammation in the right upper lobe. CT, computed tomography; GGO, ground glass opacity. 

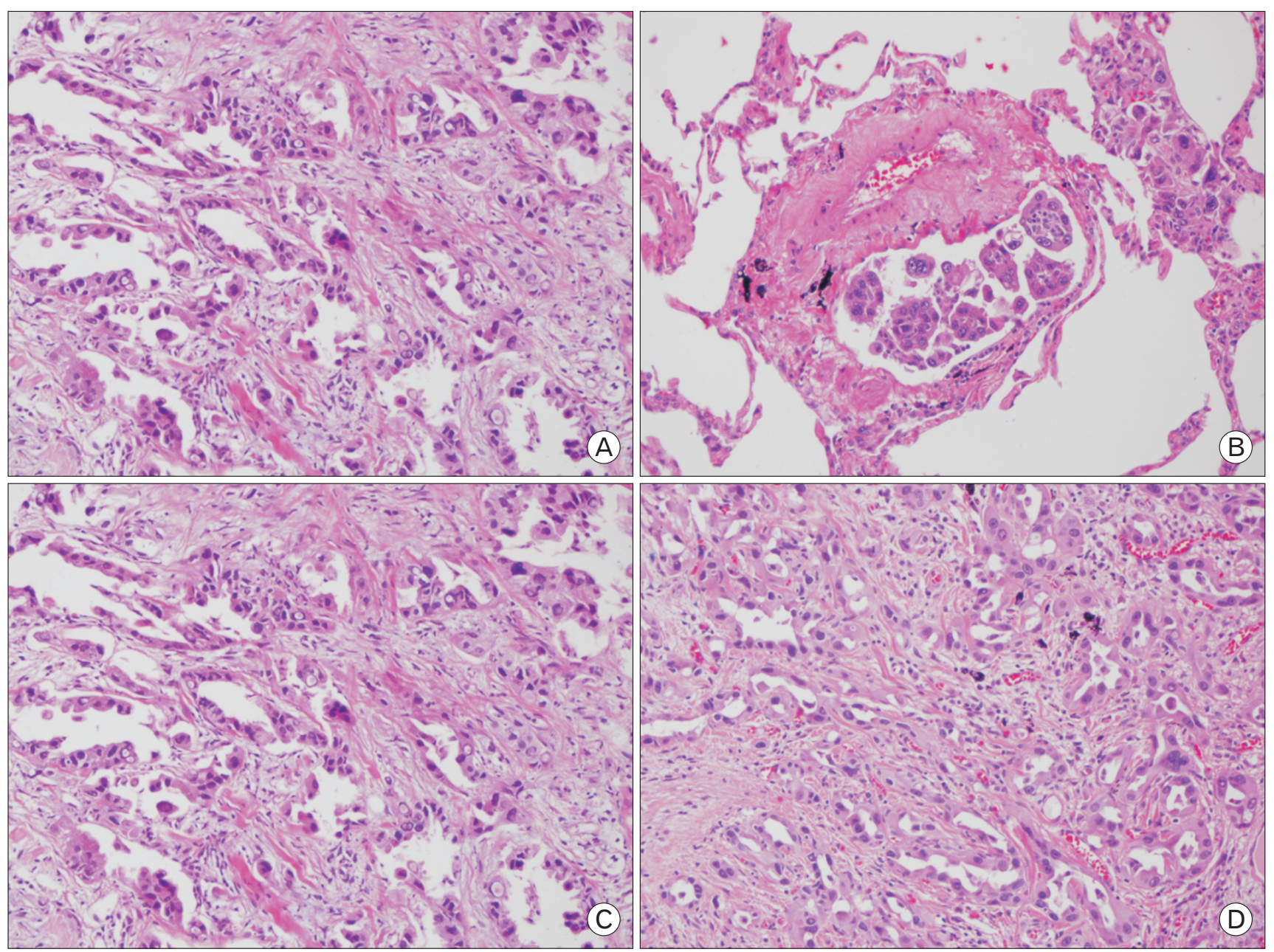

Figure 2. Tissue from the PCNA showing acinar adenocarcinoma, papillary adenocarcinoma, and lymphovascular tumor emboli. (A) H\&E, $\times 100$. (B) H\&E, $\times 200$. The morphology of the tissue from wedge resection is similar to that of the PCNA biopsy. (C) H\&E, $\times 100$. (D) H\&E, $\times 200$. PCNA, percutaneous needle aspiration.
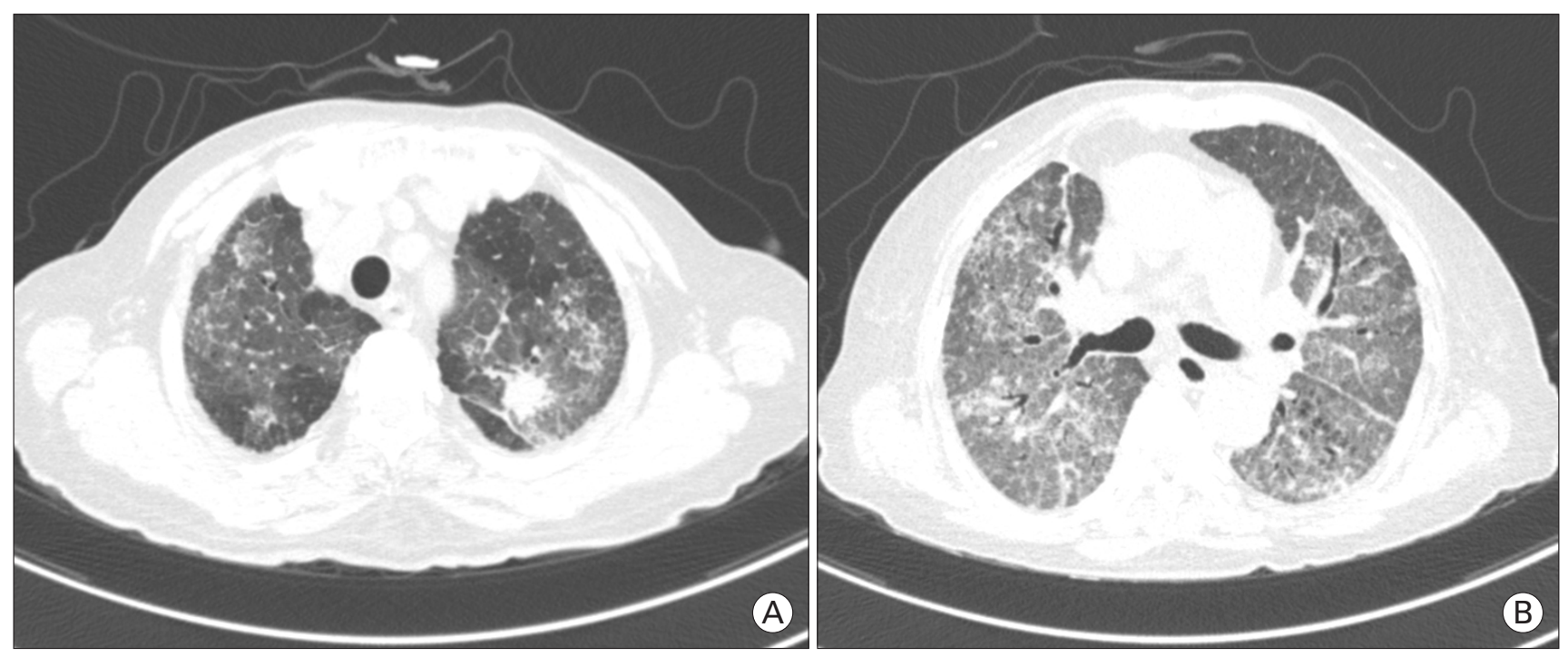

Figure 3. (A) A chest computed tomography scan showing the slight decrease of the lung cancer in the left upper lobe. (B) A chest computed tomography scan showing newly developed diffuse ground glass opacity and multifocal patchy consolidation with interstitial thickening in both the lungs. 
and sputum study tests for pneumocystis pneumonia and viral pneumonia, which are frequently found in immune-depressed patients. Moreover, pathogens such as bacteria or fungus were not detected. We planned to perform bronchoalveolar lavage, a transbronchial lung biopsy, and PCNA to exclude other infectious causes and confirm the diagnosis of acute drug-induced pneumonitis. However, the patient refused to undergo the invasive procedures. Despite the intensive care provided, which included oxygen therapy via a high-flow nasal cannula, steroid pulse therapy (methylprednisolone, $500 \mathrm{mg}$ daily for 3 days), and broad spectrum antibiotics, the patients died of hypoxia aggravation on 27 September 2015.

\section{DISCUSSION}

Lung cancer is a major cause of malignancy-associated mortality worldwide, and the 5 -year survival rate is as low as $15 \%$. Widely recognized risk factors of lung cancer include direct or indirect smoking, radon gas, and asbestos.

There is no specific treatment because most lung cancer patients are diagnosed with late stage disease. Until EGFR-TKIs were introduced, intravenous chemotherapy was the only treatment option and the average survival time was less than 12 months. The discovery of EGFR mutations and the introduction of oral EGFR-TKI agents expanded the treatment options.

EGFR is a receptor tyrosine kinase located on the cell surface. It mediates intracellular signaling and promotes cell growth, division, and migration. Activated EGFR mutations in lung adenocarcinoma have been confirmed. This mutation is more prevalent in young women, Asian people, and non-smokers. Lung adenocarcinomas with EGFR mutations are dependent on EGFR to activate intracellular signaling. When this system is blocked by EGFR-TKIs, the lung cancer cells cannot proliferate and they undergo apoptosis.

Erlotinib and gefitinib, 1st-generation EGFR-TKIs, reversibly bind to EGFR, preventing its dimerization. Most lung cancers with EGFR mutations develop resistance to the 1st-generation EGFR-TKIs within, on average, 8 to 16 months of use. The most common acquired resistance is a T790M missense mutation in exon 20 , which is reported in $50 \%$ to $60 \%$ of advanced stage patients after 1st-generation EGFR-TKI use. Afatinib, a 2nd-generation EGFR-TKI, is expected to overcome the acquired resistance that develops after 1st-generation EGFR-TKI use, by irreversibly blocking, not only EGFR, but also HER2 dimerization. Currently, there is an ongoing comparative study of 1st- and 2nd-generation EGFR-TKIs with regard to treatment efficacy in lung cancer. ${ }^{2,3)}$

Common complications associated with afatinib use include gastrointestinal and dermatologic complications such as diarrhea, vomiting, fatigue, loss of appetite, and skin rash. In addition, drug-induced pneumonitis has been reported, albeit not as frequently as with 1stgeneration EGFR-TKI use. ${ }^{2,3)}$ Acute fatal drug-induced pneumonitis was reported in several preclinical studies after treatment with erlotinib and gefitinib, and a number of meta-analyses and literature studies of drug-induced pneumonitis, including acute fatal drug-induced pneumonitis, have been conducted..$^{4-8)}$ Contrary to the erlotinib and gefitinib results, drug-induced pneumonitis, a common complication of EGFR-TKI use, had been rarely reported with afatinib use. Acute fatal pneumonitis after afatinib use, as in the current study, has been reported in only 1 previous study. ${ }^{9}$

It is yet unknown how drug-induced pneumonitis occurs after 1stgeneration EGFR-TKI use. On the basis of the results of previous studies, we have identified a possible mechanistic link between the 1stgeneration EGFR-TKI gefitinib use and drug-induced pneumonitis. ${ }^{9,10)}$ As we mentioned above, EGFR-TKIs block EGFR phosphorylation, thus preventing injured epithelium from regenerating and proliferating. ${ }^{9)}$ In addition, when an acute lung injury occurs, expression is upregulated in order to promote regeneration of the damaged epithelium. In other words, EGFR-TKIs interrupt this damage-repair mechanism of EGFR, and if these interruptions accumulate, fatal drug-induced pneumonitis may result. ${ }^{10)}$ This mechanism was identified based on gefitinib, not afatinib, use. However, afatinib and gefitinib share common characteristics, such as EGFR-blocking functions, so we assume that this potential mechanism could also apply to afatinib. We could not identify a specific and effective treatment for EGFR-TKIinduced pneumonitis. We found only 2 cases of gefitinib-induced pneumonitis that were cured by drug cessation, high dose steroids, and mechanical ventilation. ${ }^{4,5}$ These treatments were identical to those administered for general drug-induced pneumonitis. Further studies should be performed to identify the exact mechanism of, and treatment for, afatinib-induced pneumonitis.

In summary, in the treatment of metastatic lung adenocarcinoma, 2nd-generation EGFR-TKIs are expected to overcome the acquired resistance which occurs after 1st-generation EGFR-TKI use. An ongoing comparative study is evaluating the treatment efficacy of 1st- and 2ndgeneration EGFR-TKIs in lung cancer. Various target treatment agents, including EGFR-TKIs such as afatinib, have been developed and are now widely used; consequently, treatment efficacy has improved and chemotherapy is no longer the only treatment option. However, the incidence of various complications, including pulmonary toxicity, which are not widely researched, will inevitably increase. Therefore, based on the results in this case report, clinicians should be aware of the potential for afatinib treatment-associated complications.

\section{CONFLICT OF INTEREST}

No potential conflict of interest relevant to this article was reported.

\section{REFERENCES}

1. Pao W, Miller VA, Politi KA, Riely GJ, Somwar R, Zakowski MF, et al. Acquired resistance of lung adenocarcinomas to gefitinib or erlotinib is associated with a second mutation in the EGFR kinase domain. PLoS Med 2005;2:e73.

2. Soria JC, Felip E, Cobo M, Lu S, Syrigos K, Lee KH, et al. Afatinib versus erlotinib as second-line treatment of patients with advanced squa- 
mous cell carcinoma of the lung (LUX-Lung 8): an open-label randomised controlled phase 3 trial. Lancet Oncol 2015;16:897-907.

3. Yang JC, Shih JY, Su WC, Hsia TC, Tsai CM, Ou SH, et al. Afatinib for patients with lung adenocarcinoma and epidermal growth factor receptor mutations (LUX-Lung 2): a phase 2 trial. Lancet Oncol 2012; 13:539-48.

4. Zhang Y, Yang H, Zhao M, He J. Successful treatment of gefitinib-induced acute interstitial pneumonitis with corticosteroid and non-invasive BIPAP-ventilation. J Thorac Dis 2012;4:316-9.

5. Lai YC, Lin PC, Lai JI, Hsu SY, Kuo LC, Chang SC, et al. Successful treatment of erlotinib-induced acute hepatitis and acute interstitial pneumonitis with high-dose corticosteroid: a case report and literature review. Int J Clin Pharmacol Ther 2011;49:461-6.

6. Chang SC, Chang CY, Chen CY, Yu CJ. Successful erlotinib rechallenge after gefitinib-induced acute interstitial pneumonia. J Thorac Oncol
2010;5:1105-6.

7. Qi WX, Sun YJ, Shen Z, Yao Y. Risk of interstitial lung disease associated with EGFR-TKIs in advanced non-small-cell lung cancer: a metaanalysis of 24 phase III clinical trials. J Chemother 2015;27:40-51.

8. Shi L, Tang J, Tong L, Liu Z. Risk of interstitial lung disease with gefitinib and erlotinib in advanced non-small cell lung cancer: a systematic review and meta-analysis of clinical trials. Lung Cancer 2014; 83:231-9.

9. Suzuki H, Aoshiba K, Yokohori N, Nagai A. Epidermal growth factor receptor tyrosine kinase inhibition augments a murine model of pulmonary fibrosis. Cancer Res 2003;63:5054-9.

10. Ando M, Okamoto I, Yamamoto N, Takeda K, Tamura K, Seto T, et al. Predictive factors for interstitial lung disease, antitumor response, and survival in non-small-cell lung cancer patients treated with gefitinib. J Clin Oncol 2006;24:2549-56. 